\title{
Analyzing structural characteristics of object category representations from their semantic-part distributions
}

\author{
Ravi Kiran Sarvadevabhatla \\ Video Analytics Lab \\ Supercomputer Education and Research Centre \\ Indian Institute of Science, Bangalore, India \\ ravikiranessl.serc.iisc.in
}

\author{
R. Venkatesh Babu \\ Video Analytics Lab \\ Supercomputer Education and Research Centre \\ Indian Institute of Science, Bangalore, India \\ venkyeserc.iisc.in
}

\begin{abstract}
Studies from neuroscience show that part-mapping computations are employed by human visual system in the process of object recognition. In this work, we present an approach for analyzing semantic-part characteristics of $o b$ ject category representations. For our experiments, we use category-epitome, a recently proposed sketch-based spatial representation for objects. To enable part-importance analysis, we first obtain semantic-part annotations of handdrawn sketches originally used to construct the corresponding epitomes. We then examine the extent to which the semantic-parts are present in the epitomes of a category and visualize the relative importance of parts as a word cloud. Finally, we show how such word cloud visualizations provide an intuitive understanding of category-level structural trends that exist in the category-epitome object representations.
\end{abstract}

\section{Introduction}

Studies from neuroscience show that structural partmapping computations are employed by the human visual system in the process of recognition [5]. Put another way, the presence of certain parts seems to be anticipated by the visual system when it attempts to recognize an object. The knowledge of what these parts are and their relative importance for the overall task of recognition can lead to insights regarding the neuro-visual representation of objects.

In a recent work, Sarvadevabhatla et al. [7] describe the construction of sketch-based spatial representations for object categories termed category-epitomes. The epitomes are constructed to as sparse as possible while still being machine-recognizable (see Figure 2). To study these epitomes, one possibility would be to visually examine them for structural similarities on a per-category basis. However, if the number of such epitomes is large, visual ex-

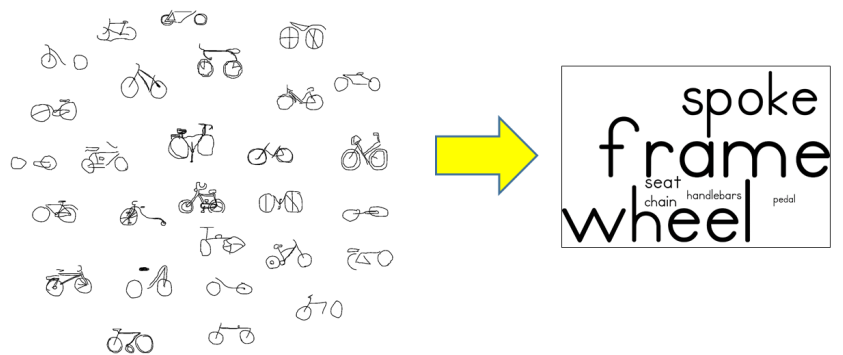

Figure 1: Sparsified yet recognizable freehand sketch representations of object category bicycle on the left. Examining such a large number of instances visually for structural similarities can be ineffective. Instead, the approach we propose captures the structurally significant parts as a semantic-part word cloud (on the right). The size of a part's name in the word cloud reflects its importance across the set of sparsified representations of the category.

amination can be ineffective. An alternate approach would be to examine the distribution of semantic-parts ${ }^{1}$ in the epitomes of each category. As we show in this work, such an approach can lead to an intuitive understanding of category-specific "signature" structural elements (parts) which persist in category-epitomes (see Figure 1). Moreover, the category-epitomes we study have been obtained using human-drawn sketches as a starting point. Therefore, our approach also creates the possibility of analyzing the underlying human neuro-visual representations as well.

\section{Related Work}

Determining the relative importance of part-level structural primitives for object category understanding has been

\footnotetext{
${ }^{1}$ E.g. spokes, seat, wheel, handle etc. are the semantic parts of a bicycle. We use the term semantic-parts to distinguish from the common interpretation of an object part as a certain spatial, unnamed portion of an object.
} 


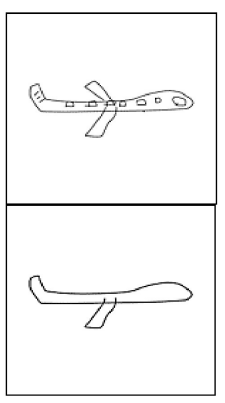

aeroplane

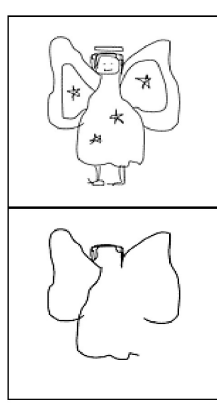

ange1

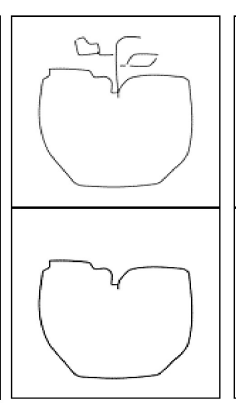

apple

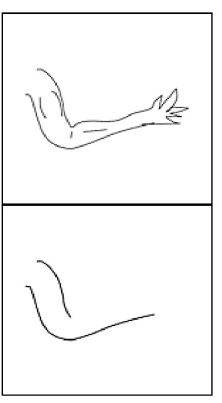

arm

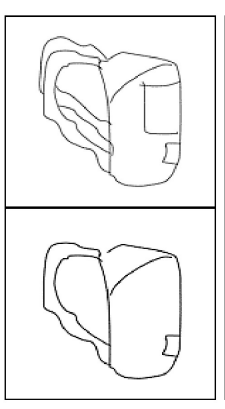

backpack

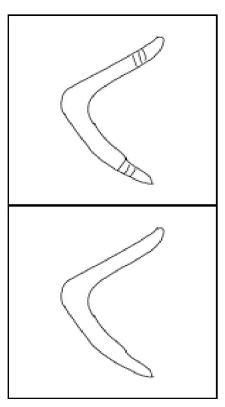

boomerang

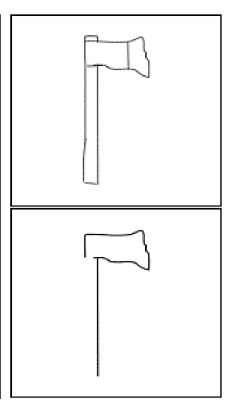

axe

Figure 2: Original sketches (top row) and corresponding category-epitomes (bottom row) for various object categories. Figure has been taken from [7].

explored only to a limited extent. Guo et al. [3] present an importance measure of shape parts based on their ability to reconstruct the whole object shape of 2-D silhouettes. However, the authors interpret parts to mean segments on the contour of the object. Ma et al. [6] propose a perception-based method to segment a sketch into semantically meaningful parts. Interestingly, they demonstrate the effectiveness of utilizing semantic parts rather than just consider parts as unnamed "regions" of the object. To the best of our knowledge, the relative importance of the semantic parts has not been studied.

\section{Construction of a part-annotated sketch database}

As the first step towards the semantic-part based understanding of category-epitomes, we manually annotated hand-drawn sketches from 13 categories $^{2}$ from the sketch database of Eitz et al. [2] for our analysis. A direction of research we intend to pursue in future involves simultaneous analysis of image and sketch based categories (whose partlevel segmentations have been provided). With this in mind, the 13 categories we examine were chosen to overlap with PASCAL-parts [1] - an image dataset containing part-level segmentations of 20 object categories. From the 20 categories in PASCAL-parts, we retained only those containing at least two dominant labeled parts. For example, category tv had only one dominant labeled part (screen) and therefore was not admissible. Within the sketches of a category, we considered only the correctly classified sketches since category-epitomes, by definition, cannot be constructed for misclassified sketches. Please refer to Section 4 of [7] for details regarding category-epitome construction.

The annotation of part contours in the sketches was performed by 10 annotators who used an annotation tool developed in-house (see Figure 3). In the figure, the

\footnotetext{
2airplane, bicycle, bus, car (sedan), cat, cow, dog, flying bird, horse, person walking, potted plant, sheep, train
}

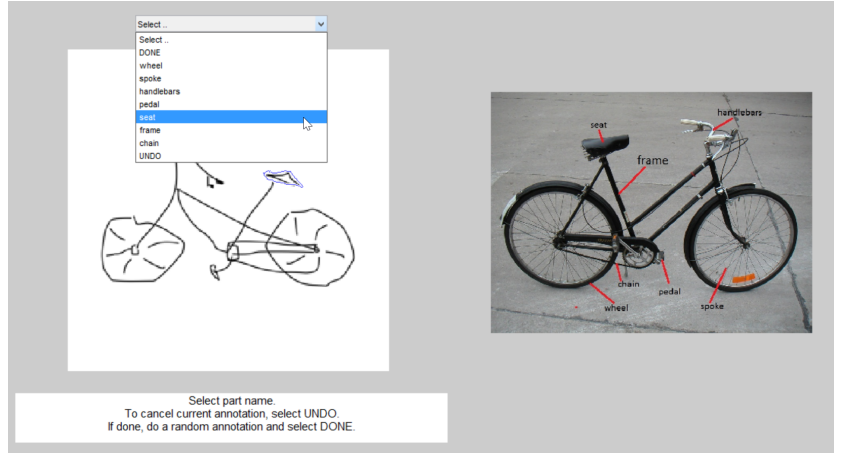

Figure 3: Screenshot of our annotation system. The sketch to be annotated and the list of parts can be seen towards the left side. The reference image to guide the annotators for part names and locations is on the right.

sketch to be annotated is on the left. As an annotation guide, a prototypical image of the category, labeled with parts, was also provided alongside the sketch. The annotators were provided basic guidelines on annotation to ensure reasonably compact boundary contours enclosing each semantic-part. At the end, we obtained semanticpart contour annotations for 283 sketches across 13 object categories for an average of 22 sketches per category. A sample annotation can be viewed in Figure 4. For each annotated sketch, the corresponding sparsified representation (category-epitome) was obtained from the epitome data provided by [7] at http: //val. serc.iisc.ernet. in/eotd/epitome_images/.

\section{Our approach}

An overview of our approach can be seen in Figure 5. In the figure, locations with numbers circled in orange correspond to important processing stages which we shall refer to in the discussion that follows. We utilize an example from the category bicycle for the purpose of illus- 


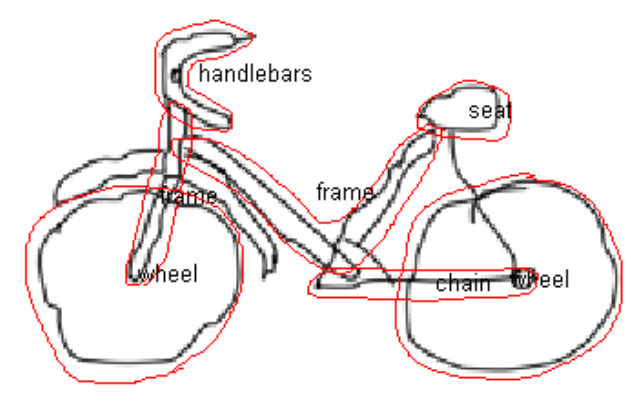

Figure 4: User annotated sketch from category bicycle

tration. Let $\mathbb{S}$ be the original full-sketch image from category $\mathcal{C}, \mathbb{E}$ its category-epitome and $\mathbb{A}$, the set of 2-D contour points which correspond to user-annotated boundaries of semantic-parts in $\mathbb{S}$. Since the category-epitome is constructed from its full-sketch counterpart, we have $\mathbb{E} \subseteq \mathbb{S}$ (i.e. the set of sketch strokes in the epitome is a subset of the strokes in the corresponding full-sketch). Let us also suppose the cardinality of $\mathbb{A}$ is $n_{a}$ and the number of semanticparts in $\mathcal{C}$ is $M$.

\subsection{Obtaining candidate part contours}

In some instances, part contours may enclose an insignificant number of pixels from epitome $\mathbb{E}$. As the first step, we filter out such contours. For each part contour, we compute the number of stroke pixels $n_{f u l l}$ in $\mathbb{S}$ that lie within the part's boundary. We also compute the number of stroke pixels $n_{e p i}$ in $\mathbb{E}$ that lie within the part's boundary. If the ratio $\frac{n_{e p i}}{n_{f u l l}}$ is larger than a threshold, the contour is added to the candidate contour list. In Figure 5, the candidate contours are shown in bold (region labeled (1)). Note that multiple occurrences of the same semantic-part type (e.g. spokes of the bicycle) which satisfy the threshold criterion are counted independently. To avoid undue importance to multiple occurrences of the same part type, we normalize by the corresponding part contour counts in full sketch to obtain a 'coarse' part-importance factor $p_{i m}$ for each semantic-part (see (2) in Figure 5). We term it a 'coarse' importance factor since it implicitly takes raw pixel counts into account for determining part importance. Shortly, we shall see how the spatial structure of the stroke is also utilized in determining the final semantic-part importance.

\subsection{Obtaining 'fine-grained' part-importance weights}

The process of annotating a part typically results in a 2D closed contour. Points on the contour tend to be in close proximity with the boundary pixels of the object. We exploit this observation to obtain a 'fine-grained' part importance factor for each part enclosed by a candidate contour.

Let $B^{(j)}=\left\{b_{i}^{(j)}=\left(x_{i}^{(j)}, y_{i}^{(j)}\right)\right\}_{i=1}^{n_{b}}, j=1 \ldots n_{a}$ be the sets of points comprising user-annotated boundary contours. Examples of such contours can be in the annotation image $\mathbb{A}$ in Figure 5 (region (3)). For a given 'part contour set' $B^{(j)}$, let $S^{(j)}=\left\{s_{k}^{(j)}=\left(x_{k}^{(j)}, y_{k}^{(j)}\right)\right\}_{k=1}^{n_{s}}, j=1 \ldots n_{a}$ be the 'full-sketch point' set of 2-D locations of stroke pixels enclosed by the part contour in the full sketch $\mathbb{S}$. Similarly, let $E^{(j)}=\left\{e_{l}^{(j)}=\left(x_{l}^{(j)}, y_{l}^{(j)}\right)\right\}_{l=1}^{n_{e}}, j=1 \ldots n_{a}$ be the 'epitome point' set of 2-D locations of stroke pixels enclosed by the part contour in epitome $\mathbb{E}$.

For each member in the 'full-sketch point' set, $s_{k}^{(j)}=$ $\left(x_{k}^{(j)}, y_{k}^{(j)}\right) \in S^{(j)}$, we find the closest-by-distance matching point $b_{i}^{(j)}=\left(x_{i}^{(j)}, y_{i}^{(j)}\right)$ from $B^{(j)}$. i.e. $b_{i}^{(j)}=$ $\operatorname{argmin}_{x} d\left(s_{k}^{(j)}, b_{x}^{(j)}\right)$. We retain only those matches $s_{k}^{(j)}, b_{i}^{(j)}$ whose distance is less than a threshold. Intuitively, this procedure enables us to identify stroke pixels of the full sketch which "hug" the candidate contour's boundary. Let $n_{\text {valid_full }}^{(j)}$ be the number of such stroke pixels $s_{k}^{(j)}$. Valid matches found using the above procedure are shown in red for a candidate part boundary in Figure 5 - refer to region (4). A similar procedure gives us $n_{\text {valid_epi }}^{(j)}$ - the number of stroke pixels in the epitome which "hug" the candidate contour's boundary (See region (5) in Figure 5). The ratio

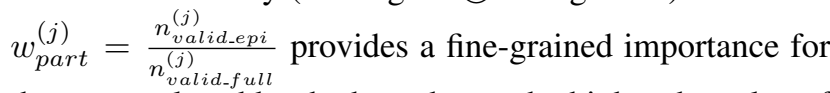
the part enclosed by the boundary - the higher the value of $w_{\text {part }}^{(j)}$, the more the number of pixels from both full sketch and the epitome which commonly "hug" the annotated part boundary. The part-importance is depicted in Figure 5 by the height of cylinders adjoining numbered regions (7), (8). As we can see, the 'wheel' is more prominently present in the epitome compared to the 'bicycle frame' thereby according the former a larger part-importance $\left(w_{\text {part }}^{(j)}\right)$ value.

\subsection{Obtaining category-wise part weights}

The above procedure of obtaining fine-grained importance weight $w_{\text {part }}^{(j)}$ is repeated for each part (indexed by $j=1,2 \ldots n_{a}$ ) enclosed by a candidate contour boundary $B^{(j)}$ by utilizing pixel location sets $S^{(j)}$ and $E^{(j)}$. These weights are combined along with the coarse importance weights $p_{i m}$ obtained in Section 4.1 to obtain the imagelevel part-wise importance weights $f_{i}, i=1,2, \ldots M$ (region labeled (6) in Figure 5). The part-wise aggregation of these weights over all the 'full sketch'-epitome pairs of a 


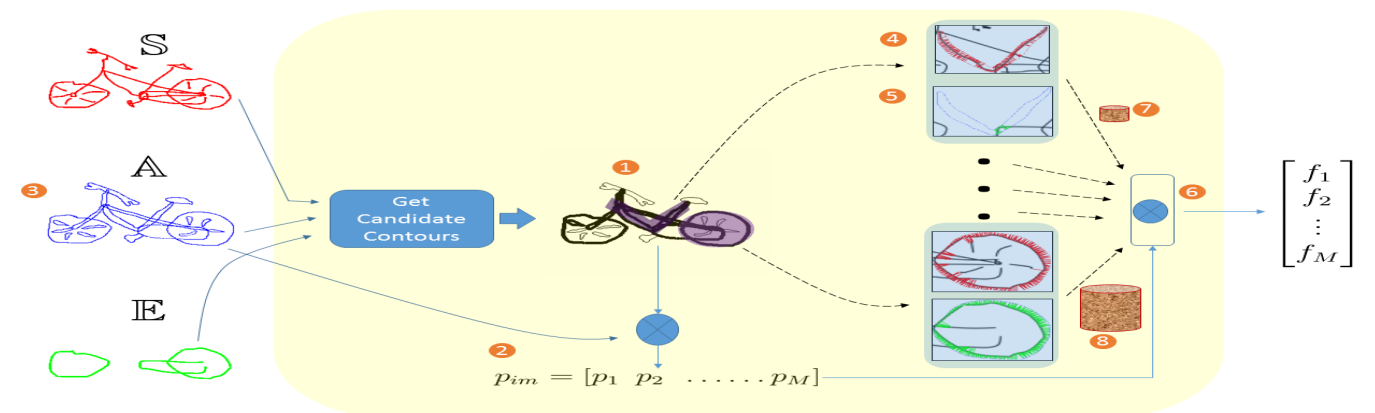

Figure 5: Determining part-importances given the full sketch $\mathbb{S}$, the corresponding category-epitome $\mathbb{E}$ and the set of user annotations $\mathbb{A}$ from the category bicycle. The output is a vector of semantic-part importances. Locations with numbered orange circles indicate key aspects of the pipeline. The sketches and annotation data have been color-coded for visualization purposes. This figure is best viewed in color.

Figure 6: Importance of semantic structural parts for object categories : Each image shows a word cloud of parts for epitomes of each category. The size of the part name indicates its relative importance across epitomes of the category. The above depictions are for LENGTH stroke sequence ordering.

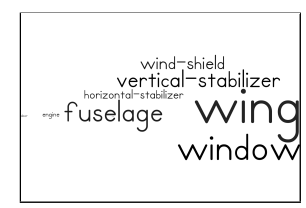

airplane

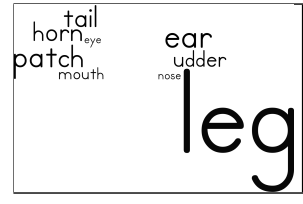

cow

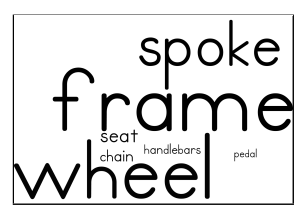

bicycle

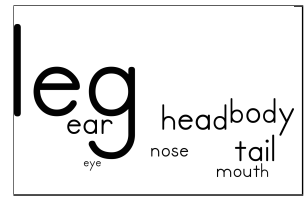

dog

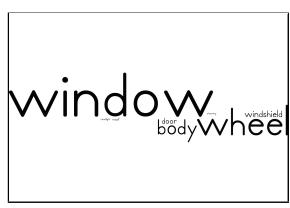

bus

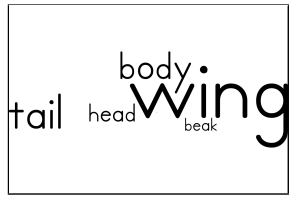

flying bird

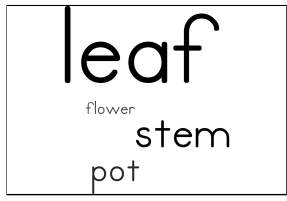

potted plant

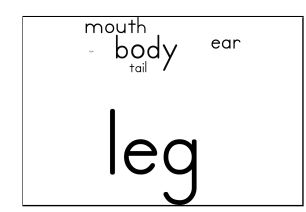

sheep

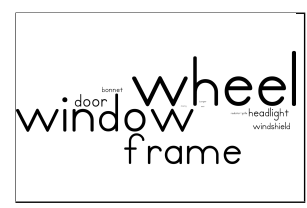

car

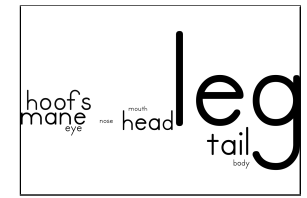

horse

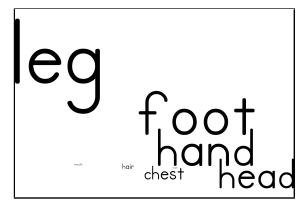

person walking

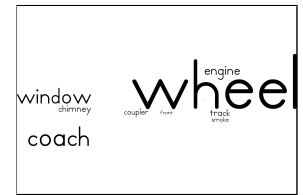

train

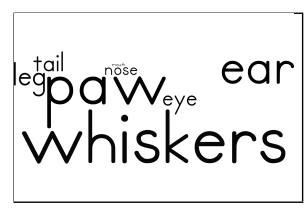

cat 

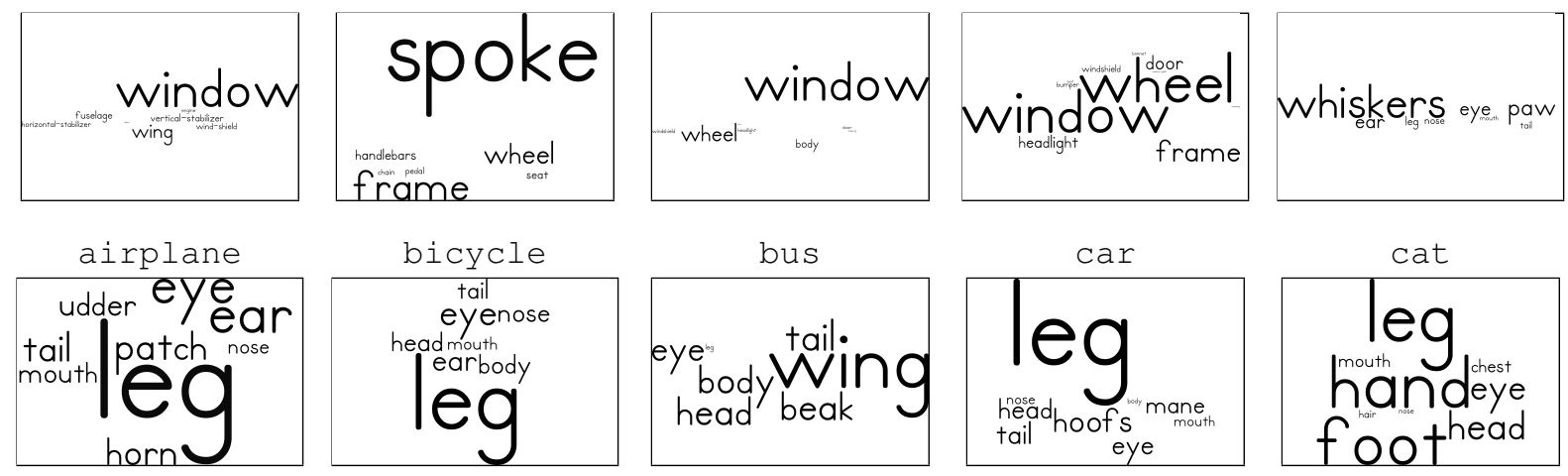

$\mathrm{COW}$

dog

flying bird

horse

person walking

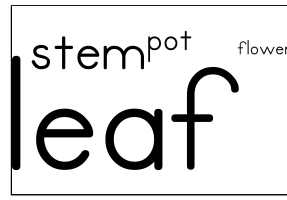

potted plant

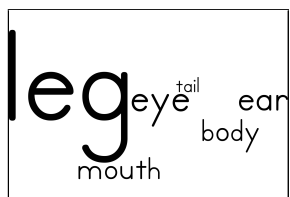

sheep

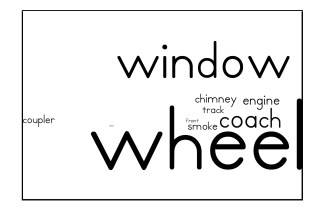

train

Figure 7: Importance of semantic structural parts for object categories : Each image shows a word cloud of parts for epitomes of each category. The size of the part name indicates its relative importance across epitomes of the category. The above depictions are for TEMPORAL stroke sequence ordering.
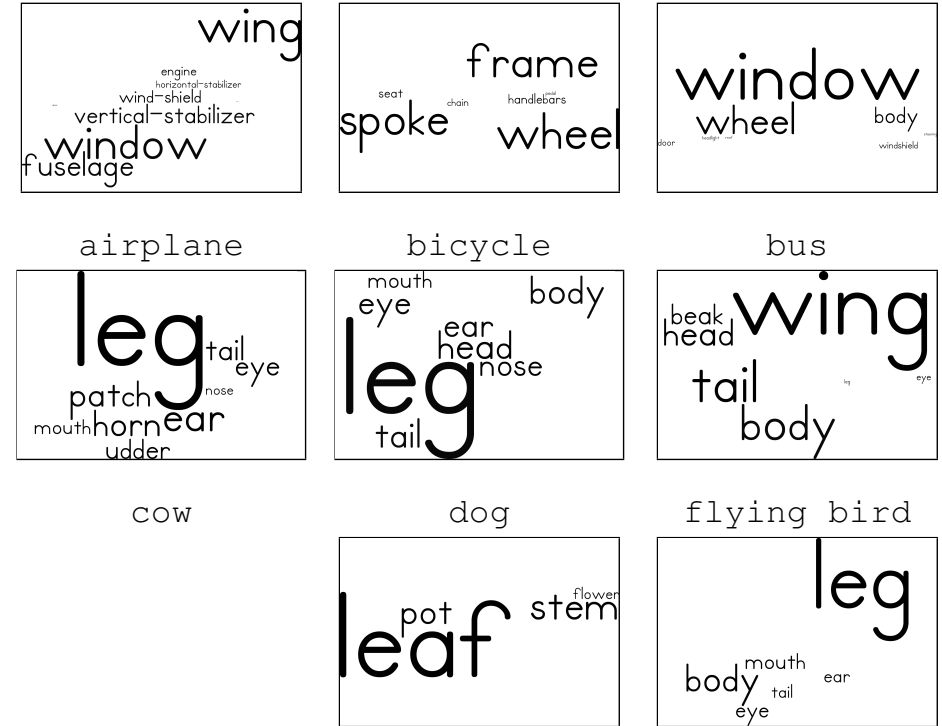

potted plant
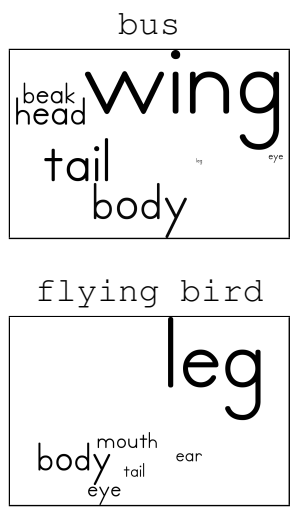

sheep
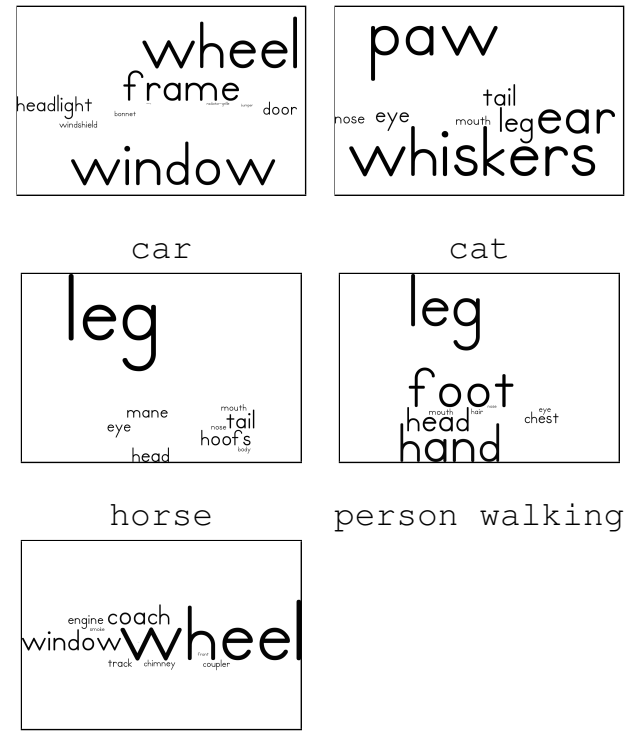

person walking

Figure 8: Importance of semantic structural parts for object categories : Each image shows a word cloud of parts for epitomes of each category. The size of the part name indicates its relative importance across epitomes of the category. The above depictions are for ALTERNATE stroke sequence ordering.

length. Essentially, for each full sketch, there can be as many category-epitomes as the number of stroke orderings.
In our analysis that follows, we keep the stroke ordering fixed over the set of categories. Details on stroke order- 
ings and their effect on category-epitomes that result can be found in Sections 4, 5 of [7].

The procedure described in Section 4 is used to generate semantic-part word clouds for the 13 object categories we have chosen. Figure 6 shows the word clouds for the LENGTH-based stroke ordering. Remember that the size of the semantic-part's name indicates its dominance in the sparsified representations (epitomes). Categories which exhibit one or two dominant parts (e.g. horse, dog, potted plant) indicate that such parts are consistently present in most of the epitomes. This, in turn, suggests a consistency in which sketches of the category are drawn. word clouds of categories with more variety in depictions (e.g. airplane, person walking) tend to contain many parts whose names are similar in size. Another interesting trend exists across semantically related categories. For instance, 'leg' is found to be the common defining signature part for the animal categories (cow, dog, horse, person walking, sheep). Similarly, for the vehicular categories (car, bus, bicycle, train), 'wheel' is a dominant part and for the flying categories (airplane, bird), 'wing' is a dominant part.

The trends mentioned above can also be seen for the TEMPORAL stroke ordering (see Figure 7). We can observe that the part importance trends are fairly same for each category across the stroke orderings. The epitomes created under TEMPORAL stroke ordering scheme tend to contain the sequence of strokes added towards the beginning. Since the part importance trends for TEMPORAL are not very dissimilar from the LENGTH-based ordering, this suggests, somewhat counter-intuitively, that people do not necessarily draw the "signature" parts of a sketch first. The ALTERNATE stroke ordering consists of an alternating combination of longest strokes and decorative strokes (temporally reversed order). However, even in this case, the essential dominance of "signature" parts remains more or less unchanged across the categories (Figure 8). These results across the stroke ordering schemes suggest that the "signature" semantic parts live up to their name - they capture the discriminative structural elements of the category and are invariant to the manner in which sketch strokes are considered in the process of epitome construction.

A more traditional, tabular version of the part word clouds with numerical values for various stroke orderings can be viewed in Tables 1, 2 and 3 .

\section{Discussion and Future Work}

In this paper, we have presented a novel framework for analyzing the structural characteristics of categoryepitomes. We have shown that semantic-part annotations of sketches can be utilized to gain an intuitive understanding of category-level and sketch-stroke-ordering level structural trends in category-epitomes. The database of part-annotated sketches of object categories is another significant contribution of our work since we can now simultaneously analyze relationships with photographic image counterparts at a semantic-part level. Finally, the word cloud based analysis we have presented is quite general and can be applied to any spatial visual object representation wherein the part labelings have been provided.

At present, we have confined our analysis to the sketch database of Eitz et al. [2]. To examine the generalizability of our approach and results, it would be interesting to apply it to the part-segmented sketch database of Huang et al. [4]. Another possible extension would be to apply the sketchpart segmentation method suggested by the aforementioned authors for the entire set of categories (instead of the 13 we have chosen) from the database of Eitz et al. [2]. 


\section{A. Pseudo-code}
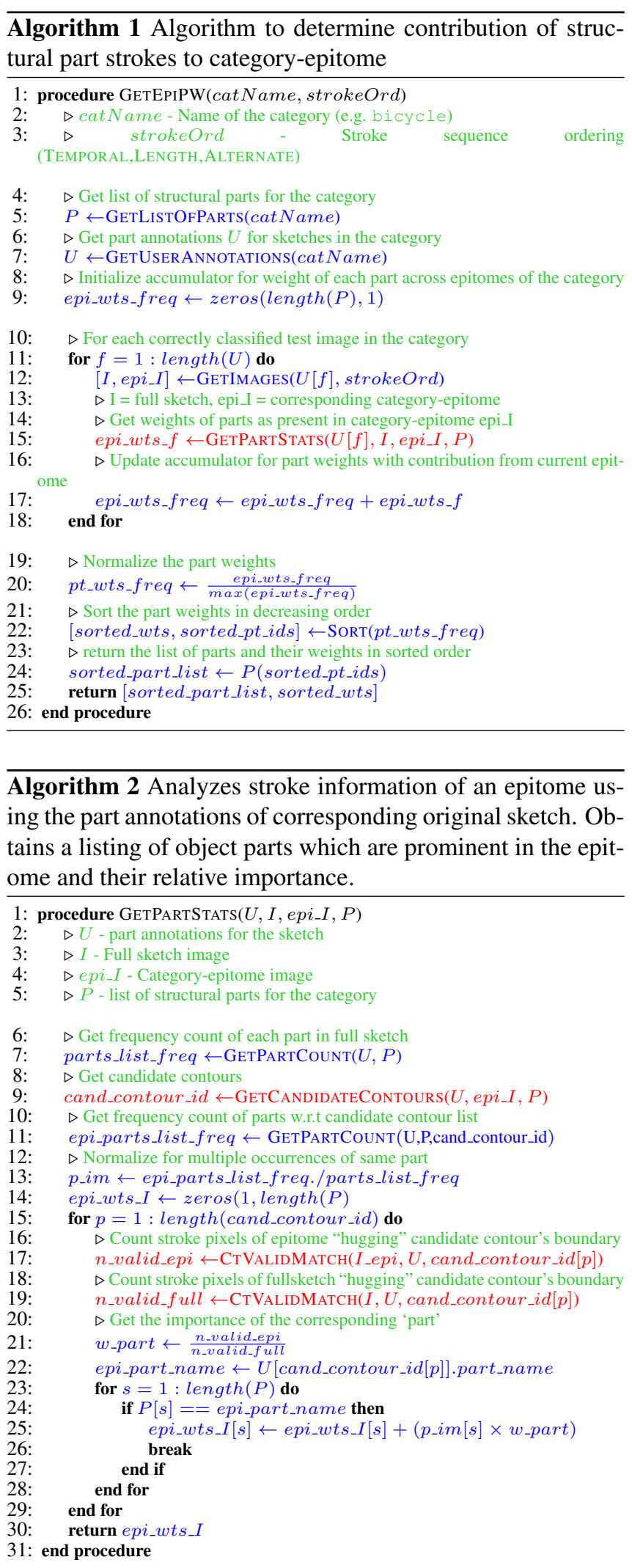
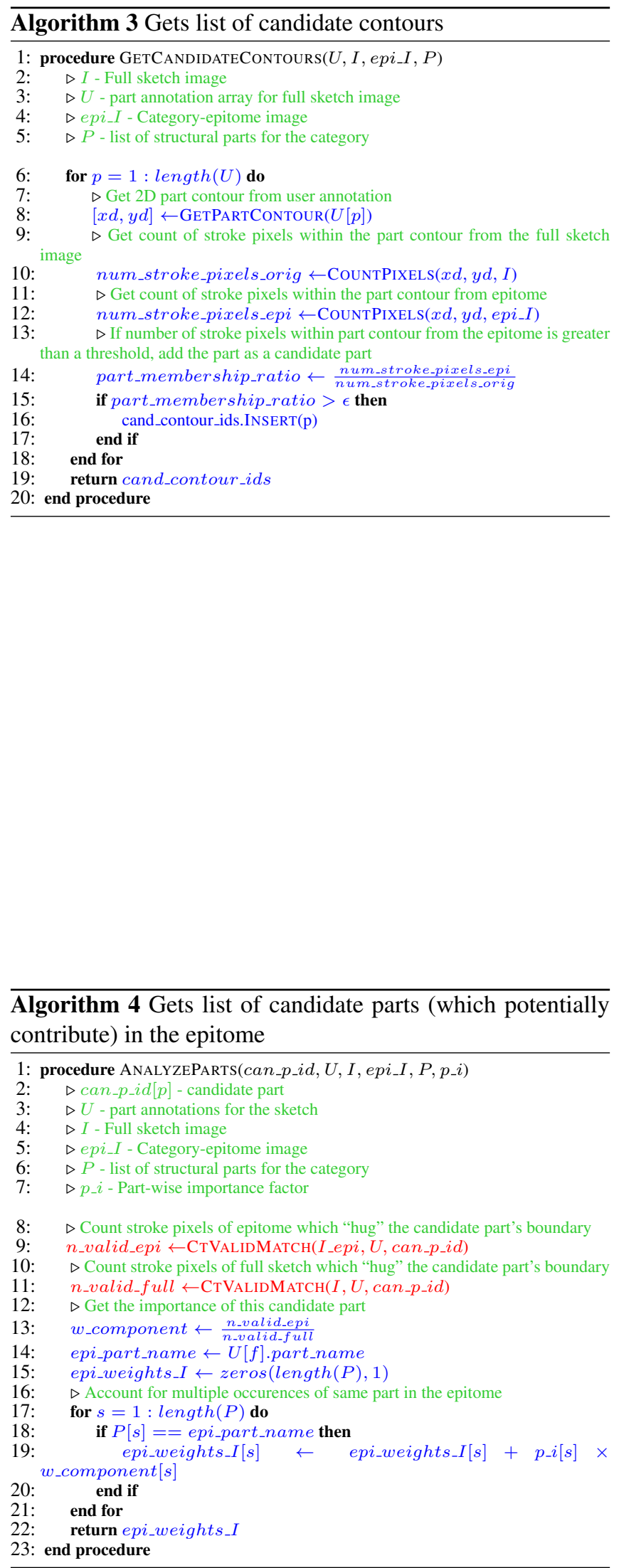


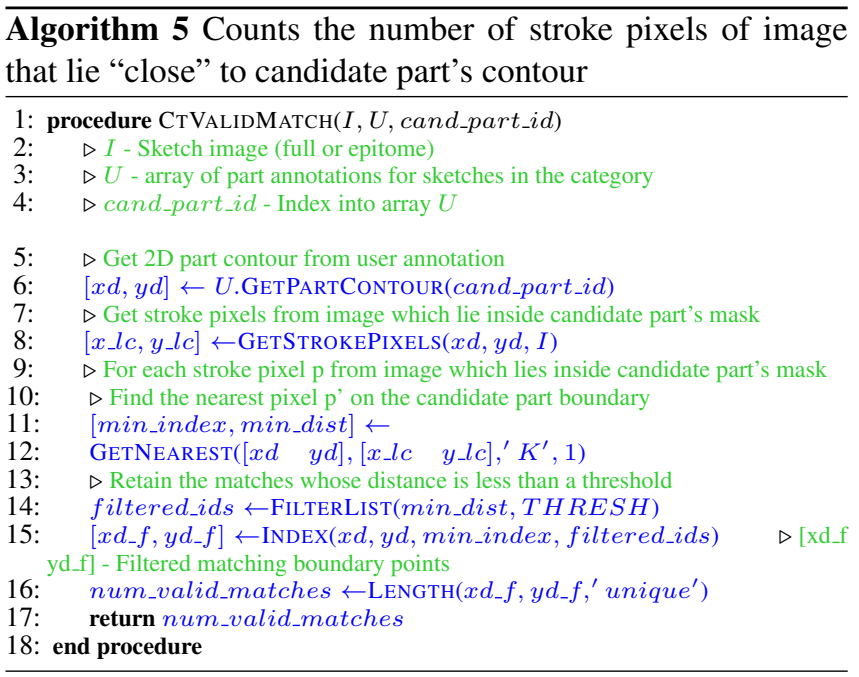




\begin{tabular}{|c|c|}
\hline Category & Epitome part-list and weights (TEMPORAL) \\
\hline airplane & $\begin{array}{l}\text { window }(1.000) \text {, wing }(0.373) \text {, fuselage }(0.190) \text {, vertical stabilizer }(0.183) \text {, wind shield }(0.159) \text {, hori- } \\
\text { zontal stabilizer }(0.151) \text {, engine }(0.095) \text {, door }(0.048) \text {, nose }(0.008)\end{array}$ \\
\hline bicycle & $\begin{array}{l}\text { spoke }(1.000) \text {, frame }(0.441) \text {, wheel }(0.304) \text {, handlebars }(0.147) \text {, seat }(0.127) \text {, pedal }(0.093) \text {, chain } \\
(0.088)\end{array}$ \\
\hline bus & $\begin{array}{l}\text { window }(1.000) \text {, wheel }(0.421) \text {, body }(0.220) \text {, windshield }(0.101) \text {, headlight }(0.094) \text {, door }(0.088) \text {, } \\
\text { steering }(0.044) \text {, roof }(0.038)\end{array}$ \\
\hline car (sedan) & $\begin{array}{l}\text { wheel }(1.000) \text {, window }(0.963) \text {, frame }(0.481) \text {, door }(0.315) \text {, headlight }(0.259) \text {, windshield }(0.148) \text {, } \\
\text { bumper }(0.111) \text {, bonnet }(0.074) \text {, seat }(0.056) \text {, steering }(0.037) \text {, radiator grille }(0.037)\end{array}$ \\
\hline cat & $\begin{array}{l}\text { whiskers }(1.000) \text {, paw }(0.531) \text {, eye }(0.449) \text {, ear }(0.449) \text {, leg }(0.245) \text {, nose }(0.224) \text {, tail }(0.204) \text {, mouth } \\
(0.143)\end{array}$ \\
\hline cow & $\begin{array}{l}\text { leg }(1.000) \text {, ear }(0.481) \text {, eye }(0.462) \text {, patch }(0.327) \text {, horn }(0.308) \text {, tail }(0.308) \text {, udder }(0.269) \text {, mouth } \\
(0.231) \text {, nose }(0.173)\end{array}$ \\
\hline $\operatorname{dog}$ & $\begin{array}{l}\text { leg (1.000), eye }(0.405) \text {, ear }(0.333) \text {, nose }(0.286) \text {, body }(0.286) \text {, head }(0.286) \text {, tail }(0.262) \text {, mouth } \\
(0.190)\end{array}$ \\
\hline flying bird & wing (1.000), beak (0.500), head (0.500), body $(0.500)$, tail $(0.500)$, eye $(0.455)$, leg $(0.091)$ \\
\hline horse & $\begin{array}{l}\text { leg }(1.000) \text {, hoofs }(0.310) \text {, eye }(0.264) \text {, head }(0.264) \text {, tail }(0.264) \text {, mane }(0.230) \text {, mouth }(0.138) \text {, nose } \\
(0.138) \text {, body }(0.069)\end{array}$ \\
\hline person walking & $\begin{array}{l}\text { leg }(1.000) \text {, hand }(0.940) \text {, foot }(0.860) \text {, head }(0.520) \text {, eye }(0.480) \text {, mouth }(0.240) \text {, chest }(0.240) \text {, hair } \\
(0.140) \text {, nose }(0.100)\end{array}$ \\
\hline potted plant & leaf (1.000), stem (0.382), pot (0.224), flower $(0.127)$ \\
\hline sheep & leg $(1.000)$, eye $(0.359)$, ear $(0.321)$, mouth $(0.269)$, body $(0.269)$, tail $(0.167)$, nose $(0.000)$ \\
\hline train & $\begin{array}{l}\text { wheel }(1.000) \text {, window }(0.578) \text {, coach }(0.311) \text {, engine }(0.156) \text {, chimney }(0.139) \text {, smoke }(0.128) \text {, coupler } \\
(0.122) \text {, track }(0.117) \text {, front }(0.061) \text {, door }(0.028)\end{array}$ \\
\hline
\end{tabular}

Table 1: Category-wise part-importances for TEMPORAL stroke ordering. Part-importances are listed in decreasing order of importance relative to the most dominant part (shown with weight 1) 


\begin{tabular}{|c|c|}
\hline Category & Epitome part-list and weights (LENGTH) \\
\hline airplane & $\begin{array}{l}\text { wing }(1.000) \text {, window }(0.692) \text {, fuselage }(0.522) \text {, vertical stabilizer }(0.423) \text {, wind shield }(0.340) \text {, hori- } \\
\text { zontal stabilizer }(0.223) \text {, engine }(0.121) \text {, door }(0.067) \text {, nose }(0.023)\end{array}$ \\
\hline bicycle & $\begin{array}{l}\text { frame }(1.000) \text {, wheel }(0.936) \text {, spoke }(0.727) \text {, seat }(0.239) \text {, chain }(0.189) \text {, handlebars }(0.149) \text {, pedal } \\
(0.124)\end{array}$ \\
\hline bus & $\begin{array}{l}\text { window }(1.000) \text {, wheel }(0.629) \text {, body }(0.346) \text {, windshield }(0.150) \text {, door }(0.132) \text {, roof }(0.063) \text {, steering } \\
(0.045) \text {, headlight }(0.044)\end{array}$ \\
\hline car (sedan) & $\begin{array}{l}\text { wheel }(1.000) \text {, window }(0.767) \text {, frame }(0.619) \text {, door }(0.254) \text {, headlight }(0.162) \text {, windshield }(0.131) \text {, } \\
\text { bonnet }(0.106) \text {, radiator grille }(0.040) \text {, bumper }(0.032) \text {, seat }(0.027) \text {, steering }(0.024)\end{array}$ \\
\hline cat & $\begin{array}{l}\text { whiskers (1.000), paw (0.867), ear }(0.717) \text {, leg }(0.394) \text {, tail }(0.334) \text {, eye }(0.317) \text {, nose }(0.232) \text {, mouth } \\
(0.066)\end{array}$ \\
\hline cow & $\begin{array}{l}\text { leg (1.000), ear }(0.327) \text {, patch }(0.299) \text {, tail }(0.261) \text {, horn }(0.258) \text {, udder }(0.220) \text {, mouth }(0.158) \text {, nose } \\
(0.114) \text {, eye }(0.110)\end{array}$ \\
\hline $\operatorname{dog}$ & $\begin{array}{l}\text { leg (1.000), head }(0.306) \text {, body }(0.297) \text {, ear }(0.290) \text {, tail }(0.287) \text {, nose }(0.173) \text {, mouth }(0.166) \text {, eye } \\
(0.104)\end{array}$ \\
\hline flying bird & wing $(1.000)$, tail $(0.549)$, body $(0.479)$, head $(0.314)$, beak $(0.225)$, leg $(0.011)$, eye $(0.000)$ \\
\hline horse & $\begin{array}{l}\text { leg (1.000), tail }(0.295) \text {, mane }(0.237) \text {, hoofs }(0.236) \text {, head }(0.232) \text {, eye }(0.097) \text {, body }(0.072) \text {, nose } \\
(0.059) \text {, mouth }(0.058)\end{array}$ \\
\hline person walking & $\begin{array}{l}\text { leg }(1.000) \text {, foot }(0.751) \text {, hand }(0.638) \text {, head }(0.507) \text {, chest }(0.233) \text {, hair }(0.094) \text {, mouth }(0.037) \text {, nose } \\
(0.030) \text {, eye }(0.005)\end{array}$ \\
\hline potted plant & leaf (1.000), stem (0.466), pot $(0.359)$, flower $(0.171)$ \\
\hline sheep & leg $(1.000)$, body $(0.401)$, mouth $(0.272)$, ear $(0.259)$, tail $(0.171)$, eye $(0.035)$, nose $(0.000)$ \\
\hline train & $\begin{array}{l}\text { wheel (1.000), coach }(0.399) \text {, window }(0.318) \text {, engine }(0.195) \text {, track }(0.141) \text {, chimney }(0.140) \text {, coupler } \\
(0.119) \text {, smoke }(0.095) \text {, front }(0.078) \text {, door }(0.013)\end{array}$ \\
\hline
\end{tabular}

Table 2: Category-wise part-importances for LENGTH stroke ordering. Part-importances are listed in decreasing order of importance relative to the most dominant part (shown with weight 1) 


\begin{tabular}{|c|c|}
\hline Category & Epitome part-list and weights (ALTERNATE) \\
\hline airplane & $\begin{array}{l}\text { wing }(1.000) \text {, window }(0.928) \text {, fuselage }(0.599) \text {, vertical stabilizer }(0.471) \text {, wind shield }(0.334) \text {, engine } \\
(0.256) \text {, horizontal stabilizer }(0.193) \text {, door }(0.054) \text {, nose }(0.027)\end{array}$ \\
\hline bicycle & $\begin{array}{l}\text { wheel }(1.000) \text {, spoke }(0.960) \text {, frame }(0.933) \text {, seat }(0.263) \text {, handlebars }(0.260) \text {, chain }(0.207) \text {, pedal } \\
(0.097)\end{array}$ \\
\hline bus & $\begin{array}{l}\text { window }(1.000) \text {, wheel }(0.567) \text {, body }(0.316) \text {, windshield }(0.130) \text {, door }(0.128) \text {, headlight }(0.065) \text {, roof } \\
(0.056) \text {, steering }(0.054)\end{array}$ \\
\hline car (sedan) & $\begin{array}{l}\text { wheel }(1.000) \text {, window }(0.935) \text {, frame }(0.651) \text {, headlight }(0.324) \text {, door }(0.281) \text {, windshield }(0.142) \text {, } \\
\text { bonnet }(0.115) \text {, radiator grille }(0.059) \text {, bumper }(0.057) \text {, steering }(0.022) \text {, seat }(0.010)\end{array}$ \\
\hline cat & $\begin{array}{l}\text { whiskers }(1.000) \text {, paw }(0.992) \text {, ear }(0.817) \text {, leg }(0.447) \text {, tail }(0.389) \text {, eye }(0.336) \text {, nose }(0.229) \text {, mouth } \\
(0.183)\end{array}$ \\
\hline cow & $\begin{array}{l}\text { leg }(1.000) \text {, ear }(0.373) \text {, horn }(0.308) \text {, patch }(0.304) \text {, tail }(0.263) \text {, eye }(0.257) \text {, udder }(0.236) \text {, mouth } \\
(0.174), \text { nose }(0.123)\end{array}$ \\
\hline $\operatorname{dog}$ & $\begin{array}{l}\text { leg (1.000), head }(0.328) \text {, body }(0.324) \text {, tail }(0.304) \text {, eye }(0.295) \text {, ear }(0.294) \text {, nose }(0.273) \text {, mouth } \\
(0.193)\end{array}$ \\
\hline flying bird & wing (1.000), tail $(0.551)$, body $(0.514)$, head $(0.387)$, beak $(0.295)$, eye $(0.102)$, leg $(0.054)$ \\
\hline horse & $\begin{array}{l}\text { leg (1.000), tail }(0.283) \text {, hoofs }(0.259) \text {, head }(0.242) \text {, mane }(0.211) \text {, eye }(0.196) \text {, mouth }(0.112) \text {, nose } \\
(0.096) \text {, body }(0.079)\end{array}$ \\
\hline person walking & $\begin{array}{l}\text { leg }(1.000) \text {, foot }(0.839) \text {, hand }(0.821) \text {, head }(0.517) \text {, chest }(0.247) \text {, mouth }(0.136) \text {, eye }(0.125) \text {, hair } \\
(0.118) \text {, nose }(0.072)\end{array}$ \\
\hline potted plant & leaf (1.000), stem (0.388), pot (0.345), flower $(0.145)$ \\
\hline sheep & leg $(1.000)$, body $(0.440)$, eye $(0.260)$, mouth $(0.251)$, ear $(0.214)$, tail $(0.195)$, nose $(0.000)$ \\
\hline train & $\begin{array}{l}\text { wheel }(1.000) \text {, window }(0.388) \text {, coach }(0.369) \text {, engine }(0.176) \text {, track }(0.153) \text {, chimney }(0.123) \text {, coupler } \\
(0.121) \text {, smoke }(0.074) \text {, front }(0.062) \text {, door }(0.011)\end{array}$ \\
\hline
\end{tabular}

Table 3: Category-wise part-importances for ALTERNATE stroke ordering. Part-importances are listed in decreasing order of importance relative to the most dominant part (shown with weight 1) 


\section{References}

[1] X. Chen, R. Mottaghi, X. Liu, S. Fidler, R. Urtasun, and A. Yuille. Detect what you can: Detecting and representing objects using holistic models and body parts. In CVPR, 2014. 2

[2] M. Eitz, J. Hays, and M. Alexa. How do humans sketch objects? SIGGRAPH, 2012. 2, 6

[3] G. Guo, Y. Wang, T. Jiang, A. L. Yuille, F. Fang, and W. Gao. A shape reconstructability measure of object part importance with applications to object detection and localization. IJCV, 2014. 2

[4] Z. Huang, H. Fu, and R. W. H. Lau. Data-driven segmentation and labeling of freehand sketches. SIGGRAPH Asia, 2014. 6

[5] A. Lovett, D. Gentner, K. Forbus, and E. Sagi. Using analogical mapping to simulate time-course phenomena in perceptual similarity. Cognitive Systems Research, 2009. 1

[6] C. Ma, Z. Dong, T. Jiang, Y. Wang, and W. Gao. A method of perceptual-based shape decomposition. In ICCV, 2013. 2

[7] R. K. Sarvadevabhatla and V. B. Radhakrishnan. Eye of the dragon : Exploring discriminatively minimalist sketchbased abstractions for object categories (url: http://val. serc.iisc.ernet.in/eotd-preprint.pdf). In ACMMM, 2015. 1, 2, 6 